\title{
Estimation in a partially linear single-index model with missing response variables and error-prone covariates
}

Xin $\mathrm{Qi}^{1,2}$ and De-Hui Wang ${ }^{1 *}$

\section{"Correspondence:}

wangdh@jlu.edu.cn

${ }^{1}$ College of Mathematics, Jilin

University, Changchun, 130012

P.R. China

Full list of author information is

available at the end of the article

\begin{abstract}
In this paper, the authors study the partially linear single-index model when the covariate $X$ is measured with additive error and the response variable $Y$ is sometimes missing. Based on the least-squared technique, an imputation method is proposed to estimate the regression coefficients, single-index coefficients, and the nonparametric function, respectively. Thereafter, asymptotical normalities of the corresponding estimators are proved. A simulation experiment and an application to a diabetes study are used to illustrate our proposed method.
\end{abstract}

Keywords: partially linear single-index model; least-squared; local linear regression; imputation estimator

\section{Introduction}

We study the partially linear single-index model

$$
Y=g\left(Z^{T} \alpha\right)+X^{T} \beta+\varepsilon
$$

where $Y$ is a response variable, $(Z, X) \in R^{p} \times R^{q}$ is covariate, $g(\cdot)$ is an unknown univariate measurable function, $\varepsilon$ is a random error with $E(\varepsilon \mid Z, X)=0, \operatorname{Var}(\varepsilon \mid Z, X)=\sigma^{2}<\infty$, and $(\alpha, \beta)$ is an unknown vector in $R^{p} \times R^{q}$ with $\|\alpha\|=1$. The restriction $\|\alpha\|=1$ ensures identifiability.

In recent years, model (1.1) has attracted broad attention because it includes two important semi-parametric models as its special cases: the single model (Ichimura [1]) and the partially linear model (Engle et al. [2]). Relevant studies about model (1.1) have been done by Carroll et al. [3], Yu et al. [4], Liang et al. [5], Xia et al. [6] and Xue et al. [7], all of which based on the complete data set.

In practice, missing-data problems are always caused by design or accident, so the statisticians, such as Liu et al. [8] and Lai et al. [9], have paid a great attention to them. Most of these researches concerning missing-data problems have been carried out on the condition that the covariates can be observed exactly. However, observations are often measured with errors, as can be seen in the papers of Liang et al. [5] and Chen et al. [10]. Nevertheless, those studies of the observations characterized by inaccurate measures are based on the complete data set. Therefore, it is necessary to study error-in-variables models with 
missing response. Taking both measurement errors in the covariates and the missing response variables into account, Liang et al. [11], Wei et al. [12] and Wei [13] have done some work in the partially linear model, in the partially linear additive model and in the partially linear varying-coefficient model, respectively.

The common method of dealing with missing data is the imputation method which was developed by Wang et al. [14] in the partially linear model. This paper, with the enlightenment of Lai $e t a l$. [15], focuses on estimating $\beta, \alpha$, and the nonparametric function $g(\cdot)$ with imputation method when the covariate $X$ is measured with additive error and the response variable $Y$ is sometimes missing in the model (1.1). It is assumed that the observation $V$ is a substitute of $X$

$$
V=X+U \text {. }
$$

The $\delta=0$ indicates that $Y$ is missing, otherwise $\delta=1$. We assume that the measurement error $U$ is independent from $(Y, Z, X, \delta)$ with $E(U)=0$ and $\operatorname{cov}(U)=\Sigma_{u u}$. At first, it is assumed that $\Sigma_{u u}$ is known. If it is unknown, it can be estimated with partial replication (Liang et al. [16]). Throughout this paper, we assume the data missing mechanism is as follows:

$$
p(\delta=1 \mid Y, Z, X)=p(\delta=1 \mid Z, X)=\pi(Z, X)
$$

for some unknown $\pi(Z, X)$. In addition, $p(\delta=1 \mid Y, Z, X, V)=\pi(Z, X)$, this is because the measurement error $U$ is independent from $(Y, Z, X, \delta)$. As is pointed out by Liang et al. [11], since $X$ is observed with measurement error, $Y$ is therefore not missing at random if no further assumptions are made.

The rest of this paper is organized as follows. In Section 2, the imputation method is used to estimate the parameters and nonparametric function. In Section 3, relative asymptotic results are presented. In Section 4, some simulation is conducted to illustrate the proposed approach, and we apply our method to analyze a diabetes data set. All proofs are shown in Section 5.

\section{Methodology}

In the following, let $\left\{\left(Y_{i}, Z_{i}, X_{i}, V_{i}, U_{i}, \delta_{i}\right), i=1,2, \ldots, n\right\}$ be independent and identically distributed, and write $A^{\otimes 2}=A \cdot A^{T}$.

\subsection{Complete method}

In order to derive the imputation estimators, first we define the complete estimators of $\beta$, $\alpha$, and the nonparametric function $g(\cdot)$. Note that $\delta_{i} Y_{i}=\delta_{i} g\left(Z_{i}^{T} \alpha\right)+\delta_{i} X_{i}^{T} \beta+\delta_{i} \varepsilon_{i}$. Taking conditional expectations given $Z^{T} \alpha$, from the assumptions, we have

$$
E\left(\delta_{i} Y_{i} \mid Z_{i}^{T} \alpha\right)=E\left(\delta_{i} \mid Z_{i}^{T} \alpha\right) g\left(Z_{i}^{T} \alpha\right)+E\left(\delta_{i} X_{i} \mid Z_{i}^{T} \alpha\right)^{T} \beta .
$$

By multiplying the both sides of model (1.1) with $E\left(\delta_{i} \mid Z_{i}^{T} \alpha\right)$, we obtain

$$
E\left(\delta_{i} \mid Z_{i}^{T} \alpha\right) Y_{i}=E\left(\delta_{i} \mid Z_{i}^{T} \alpha\right) g\left(Z_{i}^{T} \alpha\right)+E\left(\delta_{i} \mid Z_{i}^{T} \alpha\right) X_{i}^{T} \beta+E\left(\delta_{i} \mid Z_{i}^{T} \alpha\right) \varepsilon_{i} .
$$


Then making some straightforward calculations, we get

$$
\delta_{i}\left[Y_{i}-m_{2}\left(Z_{i}^{T} \alpha\right)\right]=\delta_{i}\left[X_{i}-m_{1}\left(Z_{i}^{T} \alpha\right)\right]^{T} \beta+\delta_{i} \varepsilon_{i},
$$

where $m_{1}(t)=\frac{E\left(\delta X \mid Z^{T} \alpha=t\right)}{E\left(\delta \mid Z^{T} \alpha=t\right)}, m_{2}(t)=\frac{E\left(\delta Y \mid Z^{T} \alpha=t\right)}{E\left(\delta \mid Z^{T} \alpha=t\right)}$. If $m_{1}(t), m_{2}(t)$ are known and the $X_{i}$ are observed, according to (2.1), the least-square estimator of $\beta$ can be defined as

$$
\begin{aligned}
\hat{\beta}= & \left\{\frac{1}{n} \sum_{i=1}^{n} \delta_{i}\left[X_{i}-m_{1}\left(Z_{i}^{T} \alpha\right)\right]^{\otimes 2}\right\}^{-1} \\
& \cdot\left\{\frac{1}{n} \sum_{i=1}^{n} \delta_{i}\left[X_{i}-m_{1}\left(Z_{i}^{T} \alpha\right)\right]\left[Y_{i}-m_{2}\left(Z_{i}^{T} \alpha\right)\right]\right\} .
\end{aligned}
$$

However, the $X_{i}$ are measured with error and $m_{1}\left(Z_{i}^{T} \alpha\right), m_{2}\left(Z_{i}^{T} \alpha\right)$ are unknown. From our assumptions, it follows that $E\left(\delta V \mid Z^{T} \alpha\right)=E\left(\delta X \mid Z^{T} \alpha\right)$. Therefore, the estimator of $\beta$ by the correction for the attenuation technique can be defined as

$$
\begin{aligned}
\hat{\beta}_{n}= & \left\{\frac{1}{n} \sum_{i=1}^{n} \delta_{i}\left[V_{i}-\hat{m}_{3}\left(Z_{i}^{T} \alpha\right)\right]^{\otimes 2}-\Sigma_{u u}\right\}^{-1} \\
& \cdot\left\{\frac{1}{n} \sum_{i=1}^{n} \delta_{i}\left[V_{i}-\hat{m}_{3}\left(Z_{i}^{T} \alpha\right)\right]\left[Y_{i}-\hat{m}_{2}\left(Z_{i}^{T} \alpha\right)\right]\right\},
\end{aligned}
$$

where $\hat{m}_{2}\left(Z_{i}^{T} \alpha\right)$ and $\hat{m}_{3}\left(Z_{i}^{T} \alpha\right)$ are the estimators of $m_{2}\left(Z_{i}^{T} \alpha\right)$ and $m_{3}\left(Z_{i}^{T} \alpha\right)$, respectively, and $m_{3}(t)=\frac{E\left(\delta V \mid Z^{T} \alpha=t\right)}{E\left(\delta \mid Z^{T} \alpha=t\right)}$. Let $K_{h_{1}}(t)=\frac{K_{1}\left(\frac{t}{h_{1}}\right)}{h_{1}}$, with $K_{1}(\cdot)$ being a kernel function and $h_{1}$ being a suitable bandwidth. Those estimators are defined as

$$
\hat{m}_{2}(t)=\sum_{i=1}^{n} \frac{\delta_{i} K_{h_{1}}\left(Z_{i}^{T} \alpha-t\right)}{\sum_{i=1}^{n} \delta_{i} K_{h_{1}}\left(Z_{i}^{T} \alpha-t\right)} Y_{i}, \quad \hat{m}_{3}(t)=\sum_{i=1}^{n} \frac{\delta_{i} K_{h_{1}}\left(Z_{i}^{T} \alpha-t\right)}{\sum_{i=1}^{n} \delta_{i} K_{h_{1}}\left(Z_{i}^{T} \alpha-t\right)} V_{i} .
$$

After obtaining the estimator of $\beta$, we try to estimate $g(\cdot)$ and $g^{\prime}(\cdot)$ for any fixed $\alpha$, based on $\hat{\beta}_{n}$. In fact, it becomes a single-index model which is $Y-X^{T} \beta=g\left(Z^{T} \alpha\right)+\varepsilon$. Taking conditional expectations given $Z^{T} \alpha$ on the above formula, from the previous assumptions, there is $g(t, \alpha, \beta)=E\left(Y-X^{T} \beta \mid Z^{T} \alpha=t\right)=E\left(Y-V^{T} \beta \mid Z^{T} \alpha=t\right)$. Thus, estimating $g(\cdot)$ is not necessary to be corrected. By a local linear method, we approximate $g(t)$ within the neighborhood of $t_{0}, g(t) \approx g\left(t_{0}\right)+g^{\prime}\left(t_{0}\right)\left(t-t_{0}\right)$. Then we can obtain the estimators of $g(\cdot)$ and $g^{\prime}(\cdot)$ by minimizing

$$
\min _{g\left(t_{0}\right), g^{\prime}\left(t_{0}\right)} \sum_{i=1}^{n}\left[Y_{i}-V_{i}^{T} \hat{\beta}_{n}-g\left(t_{0}\right)-g^{\prime}\left(t_{0}\right)\left(t_{i}-t_{0}\right)\right]^{2} K_{h_{2}}\left(t_{i}-t_{0}\right) \delta_{i},
$$

where $K_{h_{2}}(t)=\frac{K_{2}\left(\frac{t}{h_{2}}\right)}{h_{2}}$, with $K_{2}(\cdot)$ being a kernel function and $h_{2}$ being a suitable bandwidth. Through a direct calculation, we have

$$
\left(\begin{array}{c}
\hat{g}_{n}\left(t_{0}\right) \\
h_{2} \hat{g}_{n}^{\prime}\left(t_{0}\right)
\end{array}\right)=\frac{1}{n} \sum_{i=1}^{n}\left(\frac{1}{n} B_{0}^{T} S_{1} B_{0}\right)^{-1} B_{i 0} \delta_{i} K_{h_{2}}\left(t_{i}-t_{0}\right)\left(Y_{i}-V_{i}^{T} \hat{\beta}_{n}\right),
$$


where

$$
\begin{aligned}
& B_{i 0}=\left(\begin{array}{c}
1 \\
\frac{t_{i}-t_{0}}{h_{2}}
\end{array}\right), \quad i=1,2, \ldots, n, \quad B_{0}=\left(\begin{array}{c}
B_{10}^{T} \\
\vdots \\
B_{n 0}^{T}
\end{array}\right), \\
& S_{1}=\left(\begin{array}{ccc}
\delta_{1} K_{h_{2}}\left(t_{1}-t_{0}\right) & \\
& \ddots & \\
& & \delta_{n} K_{h_{2}}\left(t_{n}-t_{0}\right)
\end{array}\right) .
\end{aligned}
$$

In order to apply the above formulas, we have to know the estimation values of $\alpha$, which can be obtained by the following formula:

$$
\hat{\alpha}_{n}=\min _{\alpha} \sum_{i=1}^{n} \delta_{i}\left[Y_{i}-V_{i}^{T} \hat{\beta}_{n}-\hat{g}_{n}\left(Z_{i}^{T} \alpha\right)\right]^{2}
$$

The complete estimation procedure consists of the following steps:

Step 0 . Select an initial value $\hat{\alpha}_{0}$, for example, using an available method, such as the complete data estimation method proposed by Xia et al. [6], and let $\hat{\alpha}_{n}=\frac{\hat{\alpha}_{0}}{\left\|\hat{\alpha}_{0}\right\|}$.

Step 1. Based on (2.2) and (2.3), we can get $\hat{\beta}_{n k}, \hat{g}_{n k}(\cdot)$ when $\alpha=\hat{\alpha}_{n}$.

Step 2. The solution of (2.4) is written as $\hat{\alpha}_{n(k+1)}$. Let $\hat{\alpha}_{n}=\frac{\hat{\alpha}_{n(k+1)}}{\| \hat{\alpha}_{n(k+1) \|}}$.

Step 3. Iterate Steps 1 and 2 until convergence is achieved.

\subsection{Imputation method}

In this part, we will use the imputation technique to estimate $\beta, \alpha$, and the nonparametric function $g(\cdot)$. The advantage of this method is that all data can be used. First, we get $\hat{\beta}_{n}, \hat{\alpha}_{n}$, and $\hat{g}_{n}(\cdot)$ by the complete method. Let $Y_{i}^{\circ}=\delta_{i} Y_{i}+\left(1-\delta_{i}\right)\left[g\left(Z_{i}^{T} \alpha\right)+V_{i}^{T} \beta\right]$, that is, $Y_{i}^{\circ}=Y_{i}$ if $\delta_{i}=1, Y_{i}^{\circ}=g\left(Z_{i}^{T} \alpha\right)+V_{i}^{T} \beta$, otherwise. From (1.3), we have $E\left(Y^{\circ} \mid Z, X\right)=g\left(Z^{T} \alpha\right)+X^{T} \beta$. This implies

$$
Y_{i}^{\circ}=g\left(Z_{i}^{T} \alpha\right)+X_{i}^{T} \beta+e_{i}
$$

where $E\left(e_{i} \mid Z_{i}, X_{i}\right)=0$. It is just the form of the partial linear single-index model. Therefore, the least-square estimator of $\beta$ can be defined as

$$
\begin{aligned}
\breve{\beta}= & \left\{\frac{1}{n} \sum_{i=1}^{n}\left[X_{i}-E\left(X \mid Z_{i}^{T} \alpha\right)\right]^{\otimes 2}\right\}^{-1} \\
& \cdot\left\{\frac{1}{n} \sum_{i=1}^{n}\left[X_{i}-E\left(X \mid Z_{i}^{T} \alpha\right)\right]\left[Y_{i}^{\circ}-E\left(Y^{\circ} \mid Z_{i}^{T} \alpha\right)\right]\right\} .
\end{aligned}
$$

However, since the $X_{i}$ are measured with error, we cannot obtain the exact data of $Y_{i}^{\circ}$. Let $Y_{i}^{*}=\delta_{i} Y_{i}+\left(1-\delta_{i}\right)\left[\hat{g}_{n}\left(Z_{i}^{T} \hat{\alpha}_{n}\right)+V_{i}^{T} \hat{\beta}_{n}\right]$, it can be estimated as $Y_{i}^{\circ}$. Based on the correction 
for the attenuation technique, the imputation estimator of $\beta$ can be defined as

$$
\begin{aligned}
\breve{\beta}_{n}= & \left\{\frac{1}{n} \sum_{i=1}^{n}\left[V_{i}-\hat{E}\left(V \mid Z_{i}^{T} \alpha\right)\right]^{\otimes 2}-\Sigma_{\text {uu }}\right\}^{-1} \\
& \cdot\left\{\frac{1}{n} \sum_{i=1}^{n}\left[V_{i}-\hat{E}\left(V \mid Z_{i}^{T} \alpha\right)\right]\left[Y_{i}^{*}-\hat{E}\left(Y^{*} \mid Z_{i}^{T} \alpha\right)\right]-\frac{1}{n} \sum_{i=1}^{n}\left(1-\delta_{i}\right) \Sigma_{u u} \hat{\beta}_{n}\right\},
\end{aligned}
$$

where $\hat{E}\left(V \mid Z_{i}^{T} \alpha\right), \hat{E}\left(Y^{*} \mid Z_{i}^{T} \alpha\right)$ are the estimators of $E\left(V \mid Z_{i}^{T} \alpha\right), E\left(Y^{*} \mid Z_{i}^{T} \alpha\right)$, respectively. Let $K_{h_{3}}(t)=\frac{K_{3}\left(\frac{t}{h_{3}}\right)}{h_{3}}$, with $K_{3}(\cdot)$ being a kernel function and $h_{3}$ being a suitable bandwidth. Those estimators are defined as

$$
\hat{E}(V \mid t)=\sum_{i=1}^{n} \frac{K_{h_{3}}\left(Z_{i}^{T} \alpha-t\right)}{\sum_{i=1}^{n} K_{h_{3}}\left(Z_{i}^{T} \alpha-t\right)} V_{i}, \quad \hat{E}\left(Y^{*} \mid t\right)=\sum_{i=1}^{n} \frac{K_{h_{3}}\left(Z_{i}^{T} \alpha-t\right)}{\sum_{i=1}^{n} K_{h_{3}}\left(Z_{i}^{T} \alpha-t\right)} Y_{i}^{*} .
$$

Similarly, we obtain the imputation estimators of $g(t)$ and $g^{\prime}(t)$ by

$$
\min _{g\left(t_{0}\right), g^{\prime}\left(t_{0}\right)} \sum_{i=1}^{n}\left[Y_{i}^{*}-V_{i}^{T} \breve{\beta}_{n}-g\left(t_{0}\right)-g^{\prime}\left(t_{0}\right)\left(t_{i}-t_{0}\right)\right]^{2} K_{h_{4}}\left(t_{i}-t_{0}\right),
$$

where $K_{h_{4}}(t)=\frac{K_{4}\left(\frac{t}{h_{4}}\right)}{h_{4}}$, with $K_{4}(\cdot)$ being a kernel function and $h_{4}$ being a suitable bandwidth. Through a direct calculation, we have

$$
\left(\begin{array}{c}
\breve{g}_{n}\left(t_{0}\right) \\
h_{4} \breve{g}_{n}^{\prime}\left(t_{0}\right)
\end{array}\right)=\frac{1}{n} \sum_{i=1}^{n}\left(\frac{1}{n} B_{2}^{T} S_{2} B_{2}\right)^{-1} B_{i 2} K_{h_{4}}\left(t_{i}-t_{0}\right)\left(Y_{i}^{*}-V_{i}^{T} \breve{\beta}_{n}\right)
$$

where

$$
\begin{aligned}
& B_{i 2}=\left(\begin{array}{c}
1 \\
\frac{t_{i}-t_{0}}{h_{4}}
\end{array}\right), \quad i=1,2, \ldots, n, \quad B_{2}=\left(\begin{array}{c}
B_{12}^{T} \\
\vdots \\
B_{n 2}^{T}
\end{array}\right), \\
& S_{2}=\left(\begin{array}{ccc}
K_{h_{4}}\left(t_{1}-t_{0}\right) & \\
& \ddots & \\
& & K_{h_{4}}\left(t_{n}-t_{0}\right)
\end{array}\right) .
\end{aligned}
$$

As in the complete situation, if we want to use (2.6) and (2.8), it is a must to estimate $\alpha$ first, by minimizing the sum of square errors

$$
\min _{\alpha} \sum_{i=1}^{n}\left[Y_{i}^{*}-V_{i}^{T} \breve{\beta}_{n}-\breve{g}_{n}\left(Z_{i}^{T} \alpha\right)\right]^{2}
$$

say $\breve{\alpha}_{n}$. Next we do the same work as in the complete situation.

\section{Asymptotic results}

In this section, the main results of this paper are summarized. For a concise representation, let $\widetilde{\mathcal{S}}=\mathcal{S}-\frac{E\left(\delta \mathcal{S} \mid Z^{T} \alpha=t\right)}{E\left(\delta \mid Z^{T} \alpha=t\right)}$ and $\widetilde{\mathcal{S}}=\mathcal{S}-E\left(\mathcal{S} \mid Z^{T} \alpha=t\right)$, for example, $\widetilde{X}=X-\frac{E\left(\delta X \mid Z^{T} \alpha=t\right)}{E\left(\delta \mid Z^{T} \alpha=t\right)}=$ 
$X-m_{1}(t), \widetilde{\widetilde{X}}=X-E\left(X \mid Z^{T} \alpha=t\right)$. Moreover, in order to state the asymptotic results, the following assumptions will be used.

$\left(C_{1}\right)$ The matrix $\Gamma_{X \mid Z}=E\left\{\delta\left[X-m_{1}(t)\right]^{\otimes 2}\right\}$ is a positive-definite.

$\left(C_{2}\right)$ Each entry of the Hessian matrices of $m_{1}(t)$ and $m_{2}(t)$ is continuous and squared integrable, where the $(i, j)$ entry of a Hessian matrix of $g(z)$ is defined as $\frac{\partial^{2} g(z)}{\partial z_{i} \partial z_{j}}$.

$\left(C_{3}\right)$ The bandwidths are of order $n^{-\frac{1}{p+4}}$, where $p$ is the dimension of $Z$.

$\left(\mathrm{C}_{4}\right)$ The kernels $K_{i}(\cdot), i=1,2,3,4$ are a bounded symmetric density functions with compact support $[-1,1]$, and they satisfy $\int u K_{i}(u) d u=0, \int u^{2} K_{i}(u) d u \neq 0$.

$\left(C_{5}\right)$ The density function $f(t)$ of $Z^{T} \alpha$ is bounded away from 0 and has two bounded derivatives on its support.

$\left(\mathrm{C}_{6}\right) g(\cdot), m_{2}(\cdot), m_{3}(\cdot), E(V \mid \cdot), E\left(Y^{*} \mid \cdot\right)$ have two bounded, continuous derivatives on their supports.

$\left(C_{7}\right)$ The probability function $\pi(Z, X)$ has bounded continuous second partial derivatives, and is bounded away from zero on the support of $(Z, X)$.

(C) $E\left(|\varepsilon|^{4}<\infty\right), E\left(|U|^{3}<\infty\right)$.

Now we give the following asymptotical results.

Theorem 3.1 Assume that the conditions $\left(\mathrm{C}_{1}\right)-\left(\mathrm{C}_{8}\right)$ are satisfied, then we obtain

$$
\sqrt{n}\left(\breve{\beta}_{n}-\beta\right) \rightarrow N\left(0, \Sigma_{\widetilde{X}}^{-1} \Sigma_{\beta^{*}} \Sigma_{\widetilde{X}}^{-1}\right)
$$

where $\Sigma_{\widetilde{X}}=E\left\{\widetilde{\widetilde{X}}^{\otimes 2}\right\}, \Sigma_{\beta^{*}}=E\left[\left\{\left(\Gamma_{\widetilde{X}}+\Sigma_{1}-\Sigma_{2} \Gamma_{\widetilde{Z}}^{-1} \Gamma_{\widetilde{Z} \widetilde{X}}\right) \Gamma_{\widetilde{X}}^{-1} \cdot \delta\left(\widetilde{X}\left(\varepsilon-U^{T} \beta\right)+\varepsilon U-\left(U U^{T}-\right.\right.\right.\right.$ $\left.\left.\left.\left.\Sigma_{u u}\right) \beta\right)-\Sigma_{2} \Gamma_{\tilde{Z}}^{-1} \delta \widetilde{Z} g^{\prime}\left(Z^{T} \alpha\right)\left(\varepsilon-U^{T} \beta\right)\right\}^{\otimes 2}\right]$, with $\Sigma_{1}=E\left\{(1-\delta) \widetilde{X} \widetilde{X}^{T}\right\}$ and $\Sigma_{2}=E\{(1-$ $\left.\delta) \widetilde{\widetilde{X}}\left[\widetilde{Z} g^{\prime}\left(Z^{T} \alpha\right)\right]^{T}\right\}$.

Theorem 3.2 Suppose the conditions $\left(C_{1}\right)-\left(C_{8}\right)$ are satisfied, then we have

$$
\sqrt{n}\left(\breve{\alpha}_{n}-\alpha\right) \rightarrow N\left(0, \Sigma_{\widetilde{Z} \widetilde{X}}^{-1} \Sigma_{\alpha^{*}} \Sigma_{\widetilde{Z} \widetilde{X}}^{-1}\right)
$$

where $\Sigma_{\widetilde{Z} \widetilde{X}}=E\left\{\widetilde{\widetilde{Z}} \widetilde{\widetilde{X}}^{T} g^{\prime}\left(t_{0}\right)\right\}, \Sigma_{\alpha^{*}}=E\left[(Q+P)^{\otimes 2}\right]$, with $Q$ and $P$ given in (5.30) and (5.31) of Section 5, respectively.

Theorem 3.3 Suppose that the conditions $\left(\mathrm{C}_{1}\right)-\left(\mathrm{C}_{8}\right)$ hold, we have

$$
\sqrt{n h_{4}}\left(\check{g}_{n}\left(t_{0} ; \check{\alpha}_{n}, \check{\beta}_{n}\right)-g\left(t_{0}\right)\right) \rightarrow N\left(0, \frac{\mu\left(t_{0}\right) \gamma_{2}\left(K_{4}\right) \Sigma_{g}}{f\left(t_{0}\right)}\right),
$$

where $\gamma_{2}\left(K_{4}\right)=\int K_{4}^{2}(u) d u$.

\section{Numerical examples}

\subsection{Simulation}

In this subsection, we carry out some Monte Carlo experiments to show the finite sample performance of the proposed method. The set of data is generated from the following model:

$$
Y_{i}=\sin \left(\pi \cdot Z_{i}^{T} \alpha\right)+X_{i} \beta+\varepsilon_{i}, \quad V_{i}=X_{i}+U_{i}, \quad 1 \leq i \leq n,
$$


Table 1 Biases of $\alpha$ and $\beta$ under different missing functions and different sample sizes obtained by two different methods for the simulated data

\begin{tabular}{|c|c|c|c|c|c|c|c|c|c|}
\hline \multirow[b]{2}{*}{ Missing rate } & \multirow[b]{2}{*}{$n$} & \multicolumn{4}{|c|}{ Complete } & \multicolumn{4}{|c|}{ Imputation } \\
\hline & & $\hat{\alpha}_{1}$ & $\hat{\alpha}_{2}$ & $\hat{\alpha}_{3}$ & $\hat{\beta}$ & $\breve{\alpha}_{1}$ & $\breve{\alpha}_{2}$ & $\breve{\alpha}_{3}$ & $\breve{\beta}$ \\
\hline 0.30 & $\begin{array}{r}50 \\
100 \\
150\end{array}$ & $\begin{array}{l}0.0035 \\
0.0032 \\
0.0036\end{array}$ & $\begin{array}{r}0.0041 \\
-0.0018 \\
-0.0012\end{array}$ & $\begin{array}{l}-0.0057 \\
-0.0024 \\
-0.0026\end{array}$ & $\begin{array}{l}0.0308 \\
0.0230 \\
0.0234\end{array}$ & $\begin{array}{r}-0.0026 \\
0.0016 \\
0.0016\end{array}$ & $\begin{array}{r}0.0037 \\
-0.0009 \\
-0.0005\end{array}$ & $\begin{array}{l}-0.0031 \\
-0.0010 \\
-0.0011\end{array}$ & $\begin{array}{l}0.0172 \\
0.0092 \\
0.0093\end{array}$ \\
\hline 0.20 & $\begin{array}{r}50 \\
100 \\
150\end{array}$ & $\begin{array}{l}0.0033 \\
0.0030 \\
0.0031\end{array}$ & $\begin{array}{r}0.0023 \\
-0.0018 \\
-0.0011\end{array}$ & $\begin{array}{l}-0.0047 \\
-0.0023 \\
-0.0024\end{array}$ & $\begin{array}{l}0.0206 \\
0.0162 \\
0.0167\end{array}$ & $\begin{array}{r}-0.0012 \\
0.0012 \\
0.0013\end{array}$ & $\begin{array}{r}0.0009 \\
-0.0007 \\
-0.0003\end{array}$ & $\begin{array}{l}-0.0008 \\
-0.0005 \\
-0.0006\end{array}$ & $\begin{array}{l}0.0147 \\
0.0089 \\
0.0091\end{array}$ \\
\hline 0.10 & $\begin{array}{r}50 \\
100 \\
150\end{array}$ & $\begin{array}{l}0.0021 \\
0.0022 \\
0.0022\end{array}$ & $\begin{array}{r}0.0009 \\
-0.0009 \\
-0.0009\end{array}$ & $\begin{array}{l}-0.0029 \\
-0.0013 \\
-0.0015\end{array}$ & $\begin{array}{l}0.0132 \\
0.0097 \\
0.0096\end{array}$ & $\begin{array}{r}-0.0005 \\
0.0003 \\
0.0004\end{array}$ & $\begin{array}{r}0.0005 \\
-0.0002 \\
-0.0002\end{array}$ & $\begin{array}{r}0.0004 \\
-0.0004 \\
-0.0005\end{array}$ & $\begin{array}{l}0.0101 \\
0.0063 \\
0.0056\end{array}$ \\
\hline
\end{tabular}

Table 2 Standard errors of $\alpha$ and $\beta$ under different missing functions and different sample sizes obtained by two different methods for the simulated data

\begin{tabular}{|c|c|c|c|c|c|c|c|c|c|}
\hline \multirow[b]{2}{*}{ Missing rate } & \multirow[b]{2}{*}{$n$} & \multicolumn{4}{|c|}{ Complete } & \multicolumn{4}{|c|}{ Imputation } \\
\hline & & $\overline{\hat{\alpha}_{1}}$ & $\hat{\alpha}_{2}$ & $\hat{\alpha}_{3}$ & $\hat{\boldsymbol{\beta}}$ & $\breve{\alpha}_{1}$ & $\breve{\alpha}_{2}$ & $\breve{\alpha}_{3}$ & $\breve{\beta}$ \\
\hline \multirow[t]{3}{*}{0.30} & 50 & 0.1011 & 0.0883 & 0.0969 & 0.1108 & 0.0694 & 0.0586 & 0.0684 & 0.0778 \\
\hline & 100 & 0.0470 & 0.0467 & 0.0471 & 0.0662 & 0.0282 & 0.0288 & 0.0288 & 0.0448 \\
\hline & 150 & 0.0333 & 0.0331 & 0.0334 & 0.0512 & 0.0212 & 0.0215 & 0.0214 & 0.0348 \\
\hline \multirow[t]{3}{*}{0.20} & 50 & 0.0813 & 0.0782 & 0.0845 & 0.0995 & 0.0433 & 0.0416 & 0.0429 & 0.0626 \\
\hline & 100 & 0.0438 & 0.0438 & 0.0443 & 0.0601 & 0.0242 & 0.0247 & 0.0251 & 0.0369 \\
\hline & 150 & 0.0315 & 0.0315 & 0.0313 & 0.0475 & 0.0184 & 0.0191 & 0.0186 & 0.0291 \\
\hline \multirow[t]{3}{*}{0.10} & 50 & 0.0711 & 0.0731 & 0.0753 & 0.0937 & 0.0345 & 0.0327 & 0.0345 & 0.0515 \\
\hline & 100 & 0.0405 & 0.0399 & 0.0412 & 0.0560 & 0.0200 & 0.0204 & 0.0210 & 0.0306 \\
\hline & 150 & 0.0293 & 0.0290 & 0.0292 & 0.0441 & 0.0155 & 0.0159 & 0.0156 & 0.0239 \\
\hline
\end{tabular}

where $\alpha=\frac{1}{\sqrt{3}}(1,1,1)^{T}, \beta=1, X_{i} \sim N(0,1), \varepsilon_{i} \sim N(0,0.01), U_{i} \sim N(0,0.04)$, the $Z_{i}$ are trivariate with independent $U(0,1)$ components. Throughout this section, the kernel function $K_{i}(t)=\frac{15}{16}\left(1-t^{2}\right)^{2}$ if $|t| \leq 1(i=1,2,3,4)$ is used. The $h_{i}(i=1,2,3,4)$ are taken as the related bandwidths.

Based on this model, we considered the following three data missing mechanisms of the response, respectively:

Case 1. $P(\delta=1 \mid Z=z, X=x)=0.7+0.1\left(\left|z^{T} \alpha-0.5\right|+|x-1|\right)$ if $\left|z^{T} \alpha-0.5\right|+|x-1| \leq 1$, and 0.68 elsewhere;

Case 2. $P(\delta=1 \mid Z=z, X=x)=0.9-0.2\left(\left|z^{T} \alpha-0.5\right|+|x-1|\right)$ if $\left|z^{T} \alpha-0.5\right|+|x-1| \leq 1$, and 0.81 elsewhere;

Case 3. $P(\delta=1 \mid Z=z, X=x)=0.9$ for all $z$ and $x$.

The average missing rates are $0.30,0.20$, and 0.10 , respectively. For each case, we generated 1000 random samples of size $n=50,100,150$, respectively. The estimators with standard error (SE) of $\alpha$ and $\beta$ under different missing mechanisms, obtained by two different methods for the simulated data, are reported in Tables 1 and 2. The relative mean integrated square error (MISE) of $g(\cdot)$ under different missing mechanisms, obtained by two different methods for the simulated data, is reported in Table 3.

As is expected, the results fit our theory fairly well. From Tables 1 and 2, it can be seen that, for each case, the estimators of both the complete method and the imputation method close their true values, and the standard errors are small. Furthermore, the imputation estimators of $\alpha$ and $\beta$ have smaller bias and SE than the complete estimators. 
Table 3 The relative mean integrated square error of $g(\cdot)$ under different missing functions and different sample sizes obtained by two different methods for the simulated data

\begin{tabular}{|c|c|c|c|}
\hline Missing rate & $n$ & $\frac{\text { Complete }}{\hat{\boldsymbol{g}}_{n}(\cdot)}$ & $\frac{\text { Imputation }}{\breve{g}_{n}(\cdot)}$ \\
\hline 0.30 & $\begin{array}{r}50 \\
100 \\
150\end{array}$ & $\begin{array}{l}0.2014 \\
0.1251 \\
0.1014\end{array}$ & $\begin{array}{l}0.1227 \\
0.0921 \\
0.0915\end{array}$ \\
\hline 0.20 & $\begin{array}{r}50 \\
100 \\
150\end{array}$ & $\begin{array}{l}0.1514 \\
0.1102 \\
0.1026\end{array}$ & $\begin{array}{l}0.0930 \\
0.0915 \\
0.0918\end{array}$ \\
\hline 0.10 & $\begin{array}{r}50 \\
100 \\
150\end{array}$ & $\begin{array}{l}0.1451 \\
0.1138 \\
0.0996\end{array}$ & $\begin{array}{l}0.0923 \\
0.0910 \\
0.0906\end{array}$ \\
\hline
\end{tabular}

Table 4 The estimates and standard errors of $\alpha$ and $\beta$ by two different methods from the diabetes data

\begin{tabular}{llr}
\hline Parameter & Complete & \multicolumn{1}{c}{ Imputation } \\
\hline$\alpha_{1}$ & $0.0909(0.0253)$ & $0.1046(0.0251)$ \\
$\alpha_{2}$ & $0.8523(0.0356)$ & $0.8681(0.0305)$ \\
$\alpha_{3}$ & $0.5151(0.0525)$ & $0.4853(0.0530)$ \\
$\beta$ & $-1.3998(0.3201)$ & $-1.2280(0.2996)$ \\
\hline
\end{tabular}

As the sample size increases, the bias and SE of these estimators decrease for any fixed missing rate. Furthermore, as the missing rate decreases, the bias and SE of these estimators decrease for any fixed sample size. From Table 3, the imputation estimator $\breve{g}(\cdot)$ has a better performance than the complete estimator $\hat{g}(\cdot)$ in terms of MISE.

\subsection{Application to diabetes data}

In this part, we will elaborate on the proposed method through an analysis of data set from a diabetes study. Using partially linear additive model, Gai et al. [17] have analyzed the data set which includes 442 observations for diabetes patients. The response variable $Y$ is employed as a quantitative measurement of disease progression one year after baseline. The covariates include age, body mass index (BMI), average blood pressure (BP) and glucose concentration. In our notation, $Z=(\operatorname{age}, B M I, B P)^{T}, X$ is the glucose concentration measured with error. We have two replicates of $W$, the error-prone measurement of the glucose concentration, and we apply them into estimation of the measurement error variance. The precise procedures, containing the modified asymptotic variance for $\alpha$ and $\beta$, are depicted in Section 5 of Liang et al. [16]. We carry out a sensitivity analysis by taking $\sigma_{u u}=0.0161$. In order to use the data set to demonstrate our methods, we presume that $20 \%$ of the $Y$ values are missed.

The estimated values of parameters of interest via using the complete method and imputation method are presented in Table 4. It is shown that imputation estimators have smaller standard errors than complete estimators.

\section{Proofs of the main results}

In order to prove the main results, we first give some lemmas.

Lemma 5.1 Assume that the conditions $\left(\mathrm{C}_{1}\right)-\left(\mathrm{C}_{8}\right)$ hold, then we have

$$
E\left\{\hat{\varphi}\left(Z^{T} \alpha\right)-\varphi\left(Z^{T} \alpha\right)\right\}^{2}=O\left(\left(n h_{1}\right)^{-1}+h_{1}^{4}\right),
$$


where $\varphi(\cdot)$ defines one of $m_{1}(\cdot), m_{2}(\cdot), m_{3}(\cdot)$, and $\hat{\varphi}(\cdot)$ is for the estimators of $\varphi(\cdot)$.

The proof of Lemma 5.1 can be finished with the work by Mark et al. [18] and Theorems 1, 2 by Einmahl et al. [19].

Lemma 5.2 Assume that the conditions $\left(\mathrm{C}_{1}\right)-\left(\mathrm{C}_{8}\right)$ hold, then we have

$$
\sqrt{n}\left(\hat{\beta}_{n}-\beta\right) \rightarrow N\left(0, \Gamma_{\widetilde{X}}^{-1} \Sigma_{\beta} \Gamma_{\widetilde{X}}^{-1}\right)
$$

where $\Gamma_{\tilde{X}}=E\left\{\delta \widetilde{X}^{\otimes 2}\right\}, \Sigma_{\beta}=E\left\{\delta\left[\left(\varepsilon-U^{T} \beta\right) \widetilde{X}\right]^{\otimes 2}\right\}+E\left\{\delta\left[\left(U U^{T}-\Sigma_{u u}\right) \beta\right]^{\otimes 2}\right\}+E\left[\delta\left(U U^{T} \varepsilon^{2}\right)\right]$.

The proof of Lemma 5.2 is similar to the proof of Theorem 1 by Liang et al. [11]. So the details are omitted here.

Lemma 5.3 Under the conditions $\left(\mathrm{C}_{1}\right)-\left(\mathrm{C}_{8}\right)$ hold, then we have

$$
\sqrt{n}\left(\hat{\alpha}_{n}-\alpha\right) \rightarrow N\left(0, \Gamma_{\widetilde{Z}}^{-1} \Sigma_{\alpha} \Gamma_{\widetilde{Z}}^{-1}\right)
$$

where $\Gamma_{\widetilde{Z}}=E\left\{\delta\left[\widetilde{Z} g^{\prime}\left(t_{0}\right)\right]^{\otimes 2}\right\}, \Sigma_{\alpha}=E\left\{\delta\left\{\left[\widetilde{Z} g^{\prime}\left(t_{0}\right)-\Gamma_{\widetilde{Z} \widetilde{X}} \Gamma_{\widetilde{X}}^{-1} \widetilde{X}\right]\left(\varepsilon-U^{T} \beta\right)+\Gamma_{\widetilde{Z} \widetilde{X}} \Gamma_{\widetilde{X}}^{-1}\left[\left(U U^{T}-\right.\right.\right.\right.$ $\left.\left.\left.\left.\Sigma_{\text {uu }}\right) \beta-U \varepsilon\right]\right\}\right\}^{\otimes 2}$, with $\Gamma_{\tilde{Z} \tilde{X}}=E\left[\delta \widetilde{Z} \widetilde{X}^{T} g^{\prime}\left(t_{0}\right)\right]$.

The proof of Lemma 5.3 uses a similar method to the proof of Theorem 2.2 by Liang et al. [5]. Here, we only give some key steps. First, we derive the following expression:

$$
\begin{aligned}
\hat{g}_{n}\left(t_{0}, \hat{\alpha}_{n}, \hat{\beta}_{n}\right)-g\left(t_{0}\right) \\
=\frac{1}{n} \cdot \frac{1}{f\left(t_{0}\right) \mu\left(t_{0}\right)} \sum_{i=1}^{n} \delta_{i} K_{h_{2}}\left(Z_{i}^{T} \alpha-t_{0}\right)\left(\varepsilon_{i}-U_{i}^{T} \beta\right) \\
\quad-\left(\hat{\beta}_{n}-\beta\right)^{T} \frac{E\left(\delta X \mid Z^{T} \alpha=t_{0}\right)}{E\left(\delta \mid Z^{T} \alpha=t_{0}\right)}-\left(\hat{\alpha}_{n}-\alpha\right)^{T} \frac{E\left(\delta Z g^{\prime}\left(Z^{T} \alpha\right) \mid Z^{T} \alpha=t_{0}\right)}{E\left(\delta \mid Z^{T} \alpha=t_{0}\right)} \\
\quad+o_{p}\left(\frac{1}{\sqrt{n}}\right)+O_{p}\left(h_{2}^{2}\right),
\end{aligned}
$$

where $\mu\left(t_{0}\right)=E\left(\delta \mid Z^{T} \alpha=t_{0}\right)$. Then we can obtain

$$
\sqrt{n} \Gamma_{\widetilde{Z}}\left(\hat{\alpha}_{n}-\alpha\right)=\frac{1}{\sqrt{n}} \sum_{i=1}^{n} \delta_{i} g^{\prime}\left(Z_{i}^{T} \alpha\right) \widetilde{Z}_{i}\left(\varepsilon_{i}-U_{i}^{T} \beta\right)-\sqrt{n} \Gamma_{\widetilde{Z} \widetilde{X}}\left(\hat{\beta}_{n}-\beta\right)+o_{p}(1) .
$$

Combining Lemma 5.2 and the central limit theorem, we can complete the proof of Lemma 5.3.

Lemma 5.4 Suppose that the conditions $\left(\mathrm{C}_{1}\right)-\left(\mathrm{C}_{8}\right)$ hold, we have

$$
\sqrt{n h_{2}}\left(\hat{g}_{n}\left(t_{0} ; \hat{\alpha}_{n}, \hat{\beta}_{n}\right)-g\left(t_{0}\right)-\frac{1}{2} \mu_{2}\left(K_{2}\right) g^{\prime \prime}\left(t_{0}\right) h_{2}^{2}\right) \rightarrow N\left(0, \frac{\gamma_{2}\left(K_{2}\right) \Sigma_{g}}{f\left(t_{0}\right)}\right),
$$

where $\mu_{2}\left(K_{2}\right)=\int u^{2} K_{2}(u) d u, \gamma_{2}\left(K_{2}\right)=\int K_{2}^{2}(u) d u$, and $\Sigma_{g}=\sigma^{2}+\beta^{T} \Sigma_{u u} \beta$. 
Proof Note that $\hat{\alpha}_{n}-\alpha=O_{p}\left(n^{-\frac{1}{2}}\right)$, so $\hat{g}_{n}\left(t_{0} ; \hat{\alpha}_{n}, \hat{\beta}_{n}\right)-\hat{g}_{n}\left(t_{0} ; \alpha, \hat{\beta}_{n}\right)=O_{p}\left(n^{-\frac{1}{2}}\right)$. Then we only need to obtain the asymptotic expansion of $\hat{g}_{n}\left(t_{0} ; \alpha, \hat{\beta}_{n}\right)$.

From (2.3), we have

$$
\begin{aligned}
& \left(\begin{array}{c}
\hat{g}_{n}\left(t_{0} ; \alpha, \hat{\beta}_{n}\right) \\
h_{2} \hat{g}_{n}^{\prime}\left(t_{0} ; \alpha, \hat{\beta}_{n}\right)
\end{array}\right)-\left(\begin{array}{c}
g\left(t_{0}\right) \\
h_{2} g^{\prime}\left(t_{0}\right)
\end{array}\right) \\
& =\left(\frac{1}{n} B_{0}^{T} S_{1} B_{0}\right)^{-1} \frac{1}{n} \sum_{i=1}^{n} B_{i 0} \delta_{i} K_{h_{2}}\left(t_{i}-t_{0}\right) \\
& \quad \times\left\{\frac{1}{2}\left(\frac{t_{i}-t_{0}}{h_{2}}\right)^{2} g^{\prime \prime}\left(t_{0}\right) h_{2}^{2}+\left(\varepsilon_{i}-U_{i}^{T} \beta\right)-V_{i}^{T}\left(\hat{\beta}_{n}-\beta\right)+o_{p}\left(h_{2}^{2}\right)\right\} .
\end{aligned}
$$

As is pointed out by Lai et al. [15],

$$
\begin{gathered}
\frac{1}{n} B_{0}^{T} S_{1} B_{0}=\mu\left(t_{0}\right) f\left(t_{0}\right)\left(\begin{array}{cc}
1 & 0 \\
0 & \mu_{2}\left(K_{2}\right)
\end{array}\right)\left(1+o_{p}(1)\right), \\
\frac{1}{n} \sum_{i=1}^{n} B_{i 0} \delta_{i} K_{h_{2}}\left(t_{i}-t_{0}\right)\left\{\frac{1}{2}\left(\frac{t_{i}-t_{0}}{h_{2}}\right)^{2} g^{\prime \prime}\left(t_{0}\right) h_{2}^{2}\right\} \\
=\left(\begin{array}{c}
f\left(t_{0}\right) \mu\left(t_{0}\right) \frac{1}{2} \mu_{2}\left(K_{2}\right) g^{\prime \prime}\left(t_{0}\right) h_{2}^{2} \\
0
\end{array}\right)+o_{p}\left(\frac{1}{\sqrt{n h_{2}}}\right) .
\end{gathered}
$$

Combine (5.2), (5.3), and (5.4) and focus on the top equation, it follows that

$$
\begin{aligned}
\hat{g}_{n}\left(t_{0} ; \alpha, \hat{\beta}_{n}\right)-g\left(t_{0}\right) \\
=\frac{1}{2} \mu_{2}\left(K_{2}\right) g^{\prime \prime}\left(t_{0}\right) h_{2}^{2} \\
\quad+\frac{1}{n} \sum_{i=1}^{n} \frac{1}{\mu\left(t_{0}\right) f\left(t_{0}\right)} \delta_{i} K_{h_{2}}\left(t_{i}-t_{0}\right)\left[\left(\varepsilon_{i}-U_{i}^{T} \beta\right)-V_{i}^{T}\left(\hat{\beta}_{n}-\beta\right)\right]+o_{p}\left(\frac{1}{\sqrt{n h_{2}}}\right) .
\end{aligned}
$$

Because of Lemma 5.2, it is easy to obtain

$$
\frac{1}{n} \sum_{i=1}^{n} \frac{1}{\mu\left(t_{0}\right) f\left(t_{0}\right)} \delta_{i} K_{h_{2}}\left(t_{i}-t_{0}\right) V_{i}^{T}\left(\hat{\beta}_{n}-\beta\right)=o_{p}\left(\frac{1}{\sqrt{n h_{2}}}\right)
$$

then we know that

$$
\begin{aligned}
\hat{g}_{n}\left(t_{0} ; \hat{\alpha}_{n}, \hat{\beta}_{n}\right)-g\left(t_{0}\right)= & \frac{1}{2} \mu_{2}\left(K_{2}\right) g^{\prime \prime}\left(t_{0}\right) h_{2}^{2} \\
& +\frac{1}{n} \sum_{i=1}^{n} \frac{1}{\mu\left(t_{0}\right) f\left(t_{0}\right)} \delta_{i} K_{h_{2}}\left(t_{i}-t_{0}\right)\left(\varepsilon_{i}-U_{i}^{T} \beta\right)+o_{p}\left(\frac{1}{\sqrt{n h_{2}}}\right) .
\end{aligned}
$$

Applying the central limit theorem, we obtain Lemma 5.4.

Proof of Theorem 3.1 Let

$$
\triangle_{n}=\frac{1}{n} \sum_{i=1}^{n}\left\{\left[V_{i}-\hat{E}\left(V \mid Z_{i}^{T} \alpha\right)\right]^{\otimes 2}-\Sigma_{u u}\right\} .
$$


Then

$$
\triangle_{n}=E\left\{\left[X-E\left(X \mid Z^{T} \alpha\right)\right]^{\otimes 2}\right\}+o_{p}(1)=E\left\{\tilde{\widetilde{X}}^{\otimes 2}\right\}+o_{p}(1)=\Sigma_{\widetilde{X}}+o_{p}(1) .
$$

By Lemmas 5.1-5.4, it is easy to show that

$$
\begin{aligned}
\sqrt{n}\left(\check{\beta}_{n}-\beta\right)= & \triangle_{n}^{-1}\left\{\frac{1}{\sqrt{n}} \sum_{i=1}^{n}\left[\widetilde{\widetilde{V}}_{i}\left(\widetilde{\widetilde{Y}}^{*}{ }_{i}-{\widetilde{V}}_{i}^{T} \beta\right)\right]\right\} \\
& +\triangle_{n}^{-1}\left\{\sqrt{n} \Sigma_{u u} \beta-\frac{1}{\sqrt{n}} \sum_{i=1}^{n}\left(1-\delta_{i}\right) \Sigma_{u u} \hat{\beta}_{n}\right\}+o_{p}(1) .
\end{aligned}
$$

Because of the Taylor expansion and the continuity of $g^{\prime}(\cdot)$, we obtain

$$
\begin{aligned}
& \hat{g}_{n}\left(Z_{i}^{T} \hat{\alpha}_{n}\right)-g\left(Z_{i}^{T} \alpha\right) \\
& \quad=\hat{g}_{n}\left(Z_{i}^{T} \alpha\right)+g^{\prime}\left(Z_{i}^{T} \alpha\right)\left(Z_{i}^{T} \hat{\alpha}_{n}-Z_{i}^{T} \alpha\right)-g\left(Z_{i}^{T} \alpha\right)+o_{p}\left(\frac{1}{\sqrt{n}}\right) .
\end{aligned}
$$

Note that $E\left(Y^{*} \mid Z_{i}^{T} \alpha\right)=g\left(Z_{i}^{T} \alpha\right)+E\left(X \mid Z_{i}^{T} \alpha\right)^{T} \beta$. Using (5.5) yields

$$
\begin{aligned}
\left({\widetilde{Y^{*}}}_{i}-{\widetilde{V_{i}}}^{T} \beta\right)= & \left(1-\delta_{i}\right)\left[\hat{g}_{n}\left(Z_{i}^{T} \alpha\right)-g\left(Z_{i}^{T} \alpha\right)\right]+\left(1-\delta_{i}\right) g^{\prime}\left(Z_{i}^{T} \alpha\right) Z_{i}^{T}\left(\hat{\alpha}_{n}-\alpha\right) \\
& +\left(1-\delta_{i}\right) V_{i}^{T}\left(\hat{\beta}_{n}-\beta\right)+\delta_{i}\left(\varepsilon_{i}-U_{i}^{T} \beta\right)+o_{p}\left(\frac{1}{\sqrt{n}}\right) .
\end{aligned}
$$

Combining (5.1) and (5.6), and calculating directly, we have

$$
\begin{aligned}
\sqrt{n}\left(\check{\beta}_{n}-\beta\right)= & \triangle_{n}^{-1}\left\{\frac{1}{\sqrt{n}} \sum_{i=1}^{n} \widetilde{\widetilde{V}}_{i} \delta_{i}\left(\varepsilon_{i}-U_{i}^{T} \beta\right)\right\} \\
& +\triangle_{n}^{-1}\left\{\frac{1}{\sqrt{n}} \sum_{i=1}^{n} \widetilde{\widetilde{V}}_{i}\left(1-\delta_{i}\right) \widetilde{V}_{i}^{T}\left(\hat{\beta}_{n}-\beta\right)\right\} \\
& +\triangle_{n}^{-1}\left\{\frac{1}{\sqrt{n}} \sum_{i=1}^{n} \widetilde{\widetilde{V}}_{i}\left(1-\delta_{i}\right)\left[\widetilde{Z}_{i} g^{\prime}\left(Z_{i}^{T} \alpha\right)\right]^{T}\left(\hat{\alpha}_{n}-\alpha\right)\right\} \\
& +\triangle_{n}^{-1}\left\{\sqrt{n} \Sigma_{\text {uu }} \beta-\frac{1}{\sqrt{n}} \sum_{i=1}^{n}\left(1-\delta_{i}\right) \Sigma_{\text {uu }} \hat{\beta}_{n}\right\}+o_{p}(1) \\
= & \triangle_{n}^{-1}\left(I_{1}+I_{2}+I_{3}+I_{4}\right)+o_{p}(1) .
\end{aligned}
$$

By a straightforward calculation,

$$
I_{1}=\frac{1}{\sqrt{n}} \sum_{i=1}^{n}\left\{\widetilde{\widetilde{X}}_{i} \delta_{i}\left(\varepsilon_{i}-U_{i}^{T} \beta\right)+\delta_{i}\left(\varepsilon_{i} U_{i}-U_{i} U_{i}^{T} \beta\right)\right\}+o_{p}(1) .
$$

From Lemma 5.2 and the law of large numbers, it follows that

$$
I_{2}=\frac{1}{\sqrt{n}} \sum_{i=1}^{n} \widetilde{\widetilde{X}}_{i}\left(1-\delta_{i}\right) \widetilde{X}_{i}^{T}\left(\hat{\beta}_{n}-\beta\right)+\frac{1}{\sqrt{n}} \sum_{i=1}^{n}\left(1-\delta_{i}\right) \Sigma_{u u}\left(\hat{\beta}_{n}-\beta\right)+o_{p}(1)
$$




$$
\begin{aligned}
= & \sqrt{n} \Sigma_{1} \cdot \Gamma_{\tilde{X}}^{-1} \cdot \frac{1}{n} \sum_{i=1}^{n}\left\{\delta_{i}\left[\widetilde{X}_{i}\left(\varepsilon_{i}-U_{i}^{T} \beta\right)+U_{i} \varepsilon_{i}-\left(U_{i} U_{i}^{T}-\Sigma_{u u}\right) \beta\right]\right\} \\
& +\frac{1}{\sqrt{n}} \sum_{i=1}^{n}\left(1-\delta_{i}\right) \Sigma_{u u}\left(\hat{\beta}_{n}-\beta\right)+o_{p}(1) \\
= & I_{21}+I_{22}+o_{p}(1),
\end{aligned}
$$

where

$$
\Sigma_{1}=E\left\{(1-\delta) \widetilde{\widetilde{X}} \widetilde{X}^{T}\right\}
$$

Using Lemma 5.3, $I_{3}$ is decomposed as

$$
\begin{aligned}
I_{3}= & \frac{1}{\sqrt{n}} \sum_{i=1}^{n} \widetilde{\widetilde{X}}_{i}\left(1-\delta_{i}\right)\left[\widetilde{Z}_{i} g^{\prime}\left(Z_{i}^{T} \alpha\right)\right]^{T}\left(\hat{\alpha}_{n}-\alpha\right) \\
& +\frac{1}{\sqrt{n}} \sum_{i=1}^{n} U_{i}\left(1-\delta_{i}\right)\left[\widetilde{Z}_{i} g^{\prime}\left(Z_{i}^{T} \alpha\right)\right]^{T}\left(\hat{\alpha}_{n}-\alpha\right)+o_{p}(1) \\
= & \sqrt{n} \Sigma_{2} \cdot \Gamma_{\widetilde{Z}}^{-1} \cdot \frac{1}{n} \sum_{i=1}^{n} \delta_{i} \widetilde{Z}_{i} g^{\prime}\left(Z_{i}^{T} \alpha\right)\left(\varepsilon_{i}-U_{i}^{T} \beta\right)-\sqrt{n} \Sigma_{2} \cdot \Gamma_{\widetilde{Z}}^{-1} \Gamma_{\widetilde{Z} \widetilde{X}} \Gamma_{\widetilde{X}}^{-1} \\
& \cdot \frac{1}{n} \sum_{i=1}^{n}\left\{\delta_{i}\left[\widetilde{X}_{i}\left(\varepsilon_{i}-U_{i}^{T} \beta\right)+U_{i} \varepsilon_{i}-\left(U_{i} U_{i}^{T}-\Sigma_{u u}\right) \beta\right]\right\}+o_{p}(1) \\
= & I_{31}-I_{32}+o_{p}(1),
\end{aligned}
$$

where

$$
\Sigma_{2}=E\left\{(1-\delta) \widetilde{\widetilde{X}}\left[\widetilde{Z} g^{\prime}\left(Z^{T} \alpha\right)\right]^{T}\right\} .
$$

Also we have

$$
I_{4}=\sqrt{n}\left[\frac{1}{n} \sum_{i=1}^{n} \delta_{i} \Sigma_{\text {uu }} \beta-\frac{1}{n} \sum_{i=1}^{n}\left(1-\delta_{i}\right) \Sigma_{u u}\left(\hat{\beta}_{n}-\beta\right)\right] .
$$

Combining (5.7), (5.8), and (5.12), we get

$$
\begin{aligned}
I_{1} & I_{22}+I_{4} \\
& =\sqrt{n}\left[\frac{1}{n} \sum_{i=1}^{n} \delta_{i}\left\{\widetilde{\widetilde{X}}_{i}\left(\varepsilon_{i}-U_{i}^{T} \beta\right)+\varepsilon_{i} U_{i}-\left(U_{i} U_{i}^{T}-\Sigma_{u u}\right) \beta\right\}\right] \\
& =\sqrt{n}\left[\frac{1}{n} \sum_{i=1}^{n} \delta_{i}\left\{\widetilde{X}_{i}\left(\varepsilon_{i}-U_{i}^{T} \beta\right)+\varepsilon_{i} U_{i}-\left(U_{i} U_{i}^{T}-\Sigma_{u u}\right) \beta\right\}\right]+o_{p}(1) \\
& =\sqrt{n} \Gamma_{\widetilde{X}}\left(\hat{\beta}_{n}-\beta\right)+o_{p}(1) .
\end{aligned}
$$

Similarly, we obtain

$$
I_{21}-I_{32}=\left(\Sigma_{1}-\Sigma_{2} \Gamma_{\widetilde{Z}}^{-1} \Gamma_{\widetilde{Z} \widetilde{X}}\right) \Gamma_{\widetilde{X}}^{-1} \cdot \sqrt{n} \Gamma_{\widetilde{X}}\left(\hat{\beta}_{n}-\beta\right)+o_{p}(1) .
$$


To sum up,

$$
\begin{aligned}
\sqrt{n}\left(\check{\beta}_{n}-\beta\right)= & \triangle_{n}^{-1}\left(\Gamma_{\tilde{X}}+\Sigma_{1}-\Sigma_{2} \Gamma_{\widetilde{Z}}^{-1} \Gamma_{\widetilde{Z} \tilde{X}}\right) \Gamma_{\tilde{X}}^{-1} \\
& \cdot \sqrt{n}\left[\frac{1}{n} \sum_{i=1}^{n} \delta_{i}\left\{\widetilde{X}_{i}\left(\varepsilon_{i}-U_{i}^{T} \beta\right)+\varepsilon_{i} U_{i}-\left(U_{i} U_{i}^{T}-\Sigma_{u u}\right) \beta\right\}\right] \\
& -\triangle_{n}^{-1} \Sigma_{2} \Gamma_{\tilde{Z}}^{-1} \cdot \sqrt{n}\left[\frac{1}{n} \sum_{i=1}^{n} \delta_{i} g^{\prime}\left(Z_{i}^{T} \alpha\right) \widetilde{Z}_{i}\left(\varepsilon_{i}-U_{i}^{T} \beta\right)\right]+o_{p}(1) .
\end{aligned}
$$

Via the central limit theorem, Theorem 3.1 can be proved.

Proof of Theorem 3.2 We derive the following expression first:

$$
\begin{aligned}
\breve{g}_{n}\left(t_{0}, \breve{\alpha}_{n}, \breve{\beta}_{n}\right)-g\left(t_{0}\right) & \\
= & \frac{\frac{1}{n} \sum_{i=1}^{n} \delta_{i} K_{h_{4}}\left(Z_{i}^{T} \alpha-t_{0}\right)\left(\varepsilon_{i}-U_{i}^{T} \beta\right)}{\frac{1}{n} \sum_{i=1}^{n} K_{h_{4}}\left(Z_{i}^{T} \alpha-t_{0}\right)}+\left(\hat{\beta}_{n}-\beta\right)^{T} E\left[(1-\delta) X \mid Z^{T} \alpha=t_{0}\right] \\
& +\left[\hat{g}_{n}\left(t_{0}\right)-g\left(t_{0}\right)\right] \cdot\left[1-E\left(\delta \mid Z^{T} \alpha=t_{0}\right)\right]-\left(\breve{\beta}_{n}-\beta\right)^{T} E\left[X \mid Z^{T} \alpha=t_{0}\right] \\
& -\left(\breve{\alpha}_{n}-\alpha\right)^{T} E\left(Z g^{\prime}\left(Z^{T} \alpha\right) \mid Z^{T} \alpha=t_{0}\right)+o_{p}\left(\frac{1}{\sqrt{n}}\right)+O_{p}\left(h_{4}^{2}\right) .
\end{aligned}
$$

Based on (2.7), we have

$$
\begin{aligned}
0= & \frac{1}{n} \sum_{i=1}^{n} K_{h_{4}}\left(Z_{i}^{T} \breve{\alpha}_{n}-t_{0}\right)\left(\begin{array}{c}
1 \\
Z_{i}^{T} \breve{\alpha}_{n}-t_{0}
\end{array}\right) \\
& \cdot\left[Y_{i}^{*}-V_{i}^{T} \breve{\beta}_{n}-\breve{g}_{n}\left(t_{0}\right)-\breve{g}_{n}^{\prime}\left(t_{0}\right)\left(Z_{i}^{T} \breve{\alpha}_{n}-t_{0}\right)\right] .
\end{aligned}
$$

Taking only the top equation into account, using a Taylor expansion, and calculating directly, we obtain

$$
\begin{aligned}
& \frac{1}{n} \sum_{i=1}^{n} K_{h_{4}}\left(Z_{i}^{T} \alpha-t_{0}\right)\left[\breve{g}_{n}\left(t_{0}\right)-g\left(t_{0}\right)\right] \\
& =\frac{1}{n} \sum_{i=1}^{n} K_{h_{4}}\left(Z_{i}^{T} \alpha-t_{0}\right)\left[\left(1-\delta_{i}\right) V_{i}^{T}\left(\hat{\beta}_{n}-\beta\right)+\left(1-\delta_{i}\right)\left(\hat{g}_{n}\left(Z_{i}^{T} \alpha\right)-g\left(Z_{i}^{T} \alpha\right)\right)\right. \\
& \left.\quad+\delta_{i}\left(\varepsilon_{i}-U_{i}^{T} \beta\right)\right]-\left(\breve{\beta}_{n}-\beta\right)^{T} \frac{1}{n} \sum_{i=1}^{n} K_{h_{4}}\left(Z_{i}^{T} \alpha-t_{0}\right) V_{i} \\
& \quad-\left(\breve{\alpha}_{n}-\alpha\right)^{T} \frac{1}{n} \sum_{i=1}^{n} K_{h_{4}}\left(Z_{i}^{T} \alpha-t_{0}\right) Z_{i} g^{\prime}\left(t_{0}\right)+o_{p}\left(\frac{1}{\sqrt{n}}\right)+O_{p}\left(h_{4}^{2}\right) .
\end{aligned}
$$

Dividing all terms in (5.15) by $\frac{1}{n} \sum_{i=1}^{n} K_{h_{4}}\left(Z_{i}^{T} \alpha-t_{0}\right)$, we have

$$
\begin{aligned}
\breve{g}_{n}\left(t_{0}\right)-g\left(t_{0}\right)= & \frac{\frac{1}{n} \sum_{i=1}^{n} \delta_{i} K_{h_{4}}\left(Z_{i}^{T} \alpha-t_{0}\right)\left(\varepsilon_{i}-U_{i}^{T} \beta\right)}{\frac{1}{n} \sum_{i=1}^{n} K_{h_{4}}\left(Z_{i}^{T} \alpha-t_{0}\right)} \\
& +\left(\hat{\beta}_{n}-\beta\right)^{T} \frac{\frac{1}{n} \sum_{i=1}^{n}\left(1-\delta_{i}\right) K_{h_{4}}\left(Z_{i}^{T} \alpha-t_{0}\right) V_{i}}{\frac{1}{n} \sum_{i=1}^{n} K_{h_{4}}\left(Z_{i}^{T} \alpha-t_{0}\right)}
\end{aligned}
$$




$$
\begin{aligned}
& +\left(\hat{g}_{n}\left(t_{0}\right)-g\left(t_{0}\right)\right) \frac{\frac{1}{n} \sum_{i=1}^{n}\left(1-\delta_{i}\right) K_{h_{4}}\left(Z_{i}^{T} \alpha-t_{0}\right)}{\frac{1}{n} \sum_{i=1}^{n} K_{h_{4}}\left(Z_{i}^{T} \alpha-t_{0}\right)} \\
& -\left(\breve{\beta}_{n}-\beta\right)^{T} \frac{\frac{1}{n} \sum_{i=1}^{n} K_{h_{4}}\left(Z_{i}^{T} \alpha-t_{0}\right) V_{i}}{\frac{1}{n} \sum_{i=1}^{n} K_{h_{4}}\left(Z_{i}^{T} \alpha-t_{0}\right)} \\
& -\left(\breve{\alpha}_{n}-\alpha\right)^{T} \frac{\frac{1}{n} \sum_{i=1}^{n} K_{h_{4}}\left(Z_{i}^{T} \alpha-t_{0}\right) Z_{i} g^{\prime}\left(t_{0}\right)}{\frac{1}{n} \sum_{i=1}^{n} K_{h_{4}}\left(Z_{i}^{T} \alpha-t_{0}\right)}+o_{p}\left(\frac{1}{\sqrt{n}}\right)+O_{p}\left(h_{4}^{2}\right) .
\end{aligned}
$$

Note that

$$
\begin{aligned}
& \frac{\frac{1}{n} \sum_{i=1}^{n}\left(1-\delta_{i}\right) K_{h_{4}}\left(Z_{i}^{T} \alpha-t_{0}\right) V_{i}}{\frac{1}{n} \sum_{i=1}^{n} K_{h_{4}}\left(Z_{i}^{T} \alpha-t_{0}\right)}=E\left[(1-\delta) X \mid Z^{T} \alpha=t_{0}\right]\left(1+o_{p}(1)\right), \\
& \frac{\frac{1}{n} \sum_{i=1}^{n}\left(1-\delta_{i}\right) K_{h_{4}}\left(Z_{i}^{T} \alpha-t_{0}\right)}{\frac{1}{n} \sum_{i=1}^{n} K_{h_{4}}\left(Z_{i}^{T} \alpha-t_{0}\right)}=1-E\left(\delta \mid Z^{T} \alpha=t_{0}\right)\left(1+o_{p}(1)\right), \\
& \frac{\frac{1}{n} \sum_{i=1}^{n} K_{h_{4}}\left(Z_{i}^{T} \alpha-t_{0}\right) V_{i}}{\frac{1}{n} \sum_{i=1}^{n} K_{h_{4}}\left(Z_{i}^{T} \alpha-t_{0}\right)}=E\left(X \mid Z^{T} \alpha=t_{0}\right)\left(1+o_{p}(1)\right),
\end{aligned}
$$

and

$$
\frac{\frac{1}{n} \sum_{i=1}^{n} K_{h_{4}}\left(Z_{i}^{T} \alpha-t_{0}\right) Z_{i} g^{\prime}\left(t_{0}\right)}{\frac{1}{n} \sum_{i=1}^{n} K_{h_{4}}\left(Z_{i}^{T} \alpha-t_{0}\right)}=E\left(Z g^{\prime}\left(Z^{T} \alpha\right) \mid Z^{T} \alpha=t_{0}\right)\left(1+o_{p}(1)\right) .
$$

Thus, equation (5.14) follows.

Second, we give the proof of Theorem 3.2. From (2.9), $\breve{\alpha}_{n}$ is the solution of

$$
\frac{1}{n} \sum_{i=1}^{n}\left[Y_{i}^{*}-V_{i}^{T} \breve{\beta}_{n}-\breve{g}_{n}\left(Z_{i}^{T} \breve{\alpha}_{n}\right)\right] \cdot \breve{g}_{n}^{\prime}\left(Z_{i}^{T} \breve{\alpha}_{n}\right) Z_{i}=0,
$$

it can be rewritten as

$$
\begin{aligned}
& \frac{1}{n} \sum_{i=1}^{n} g^{\prime}\left(Z_{i}^{T} \alpha\right) Z_{i}\left\{\left[Y_{i}^{*}-V_{i}^{T} \beta-g\left(Z_{i}^{T} \alpha\right)\right]-\left[\breve{g}_{n}\left(Z_{i}^{T} \breve{\alpha}_{n}\right)-g\left(Z_{i}^{T} \alpha\right)\right]\right. \\
& \left.\quad-V_{i}^{T}\left(\breve{\beta}_{n}-\beta\right)\right\} \cdot\left(1+o_{p}(1)\right)=0 .
\end{aligned}
$$

Because of the Taylor expansion and the continuity of $g^{\prime}(\cdot)$, we can obtain

$$
\begin{aligned}
& \breve{g}_{n}\left(Z_{i}^{T} \breve{\alpha}_{n}\right)-g\left(Z_{i}^{T} \alpha\right) \\
& \quad=\breve{g}_{n}\left(Z_{i}^{T} \alpha\right)+g^{\prime}\left(Z_{i}^{T} \alpha\right)\left(Z_{i}^{T} \breve{\alpha}_{n}-Z_{i}^{T} \alpha\right)-g\left(Z_{i}^{T} \alpha\right)+o_{p}\left(\frac{1}{\sqrt{n}}\right) .
\end{aligned}
$$

By (5.17), (5.16) can be written as

$$
\begin{aligned}
& \frac{1}{n} \sum_{i=1}^{n} g^{\prime}\left(Z_{i}^{T} \alpha\right) Z_{i}\left\{\delta_{i}\left(\varepsilon_{i}-U_{i}^{T} \beta\right)+\left(1-\delta_{i}\right) V_{i}^{T}\left(\hat{\beta}_{n}-\beta\right)\right. \\
& \quad+\left(1-\delta_{i}\right)\left[\hat{g}_{n}\left(Z_{i}^{T} \alpha\right)-g\left(Z_{i}^{T} \alpha\right)\right]-\left[\breve{g}_{n}\left(Z_{i}^{T} \alpha\right)-g\left(Z_{i}^{T} \alpha\right)\right]-V_{i}^{T}\left(\breve{\beta}_{n}-\beta\right) \\
& \left.\quad-g^{\prime}\left(Z_{i}^{T} \alpha\right) Z_{i}^{T}\left(\breve{\alpha}_{n}-\alpha\right)\right\}\left(1+o_{p}(1)\right)=0
\end{aligned}
$$


Applying (5.14) to the equation, it is easy to obtain

$$
\begin{aligned}
\frac{1}{\sqrt{n}} \sum_{i=1}^{n} \delta_{i} g^{\prime}\left(Z_{i}^{T} \alpha\right) Z_{i}\left(\varepsilon_{i}-U_{i}^{T} \beta\right) & \\
& -\frac{1}{\sqrt{n}} \sum_{i=1}^{n} g^{\prime}\left(Z_{i}^{T} \alpha\right) Z_{i} \cdot \frac{\frac{1}{n} \sum_{j=1}^{n} \delta_{j} K_{h_{4}}\left(Z_{j}^{T} \alpha-Z_{i}^{T} \alpha\right)\left(\varepsilon_{j}-U_{j}^{T} \beta\right)}{\frac{1}{n} \sum_{j=1}^{n} K_{h_{4}}\left(Z_{j}^{T} \alpha-Z_{i}^{T} \alpha\right)} \\
& -\frac{1}{\sqrt{n}} \sum_{i=1}^{n} g^{\prime}\left(Z_{i}^{T} \alpha\right) Z_{i}\left[\hat{g}_{n}\left(Z_{i}^{T} \alpha\right)-g\left(Z_{i}^{T} \alpha\right)\right] \cdot\left[\delta_{i}-E\left(\delta \mid Z^{T} \alpha=Z_{i}^{T} \alpha\right)\right] \\
& +\left(\hat{\beta}_{n}-\beta\right)^{T} \frac{1}{\sqrt{n}} \sum_{i=1}^{n} g^{\prime}\left(Z_{i}^{T} \alpha\right) Z_{i}\left[\left(1-\delta_{i}\right) V_{i}-E\left((1-\delta) V \mid Z^{T} \alpha=Z_{i}^{T} \alpha\right)\right] \\
= & \frac{1}{\sqrt{n}} \sum_{i=1}^{n} g^{\prime}\left(Z_{i}^{T} \alpha\right) Z_{i}\left(\begin{array}{c}
\widetilde{\widetilde{Z}}_{i} g^{\prime}\left(Z_{i}^{T} \alpha\right) \\
\tilde{\widetilde{X}}_{i}+U_{i}
\end{array}\right)^{T}\left(\begin{array}{c}
\breve{\alpha}_{n}-\alpha \\
\breve{\beta}_{n}-\beta
\end{array}\right)+o_{p}(1) .
\end{aligned}
$$

Note that the second term of the left-hand side of (5.18) is

$$
\frac{1}{\sqrt{n}} \sum_{i=1}^{n} \delta_{i}\left(\varepsilon_{i}-U_{i}^{T} \beta\right) E\left[Z g^{\prime}\left(Z^{T} \alpha\right) \mid Z^{T} \alpha=Z_{i}^{T} \alpha\right]+o_{p}(1)
$$

Then the first two terms of the left-hand side of (5.18) are as follows:

$$
\begin{aligned}
\frac{1}{\sqrt{n}} \sum_{i=1}^{n} \delta_{i} g^{\prime}\left(Z_{i}^{T} \alpha\right) Z_{i}\left(\varepsilon_{i}-U_{i}^{T} \beta\right) \\
\quad-\frac{1}{\sqrt{n}} \sum_{i=1}^{n} \delta_{i}\left(\varepsilon_{i}-U_{i}^{T} \beta\right) E\left[Z g^{\prime}\left(Z^{T} \alpha\right) \mid Z^{T} \alpha=Z_{i}^{T} \alpha\right] \\
=\frac{1}{\sqrt{n}} \sum_{i=1}^{n} \delta_{i}\left(\varepsilon_{i}-U_{i}^{T} \beta\right) \widetilde{Z}_{i} g^{\prime}\left(Z_{i}^{T} \alpha\right) .
\end{aligned}
$$

Applying (5.1) to the third term of the left-hand side of (5.18), it follows that

$$
\begin{aligned}
\frac{1}{\sqrt{n}} \sum_{i=1}^{n} g^{\prime}\left(Z_{i}^{T} \alpha\right) Z_{i}\left[\delta_{i}-E\left(\delta \mid Z^{T} \alpha=Z_{i}^{T} \alpha\right)\right] \cdot\left[\hat{g}_{n}\left(Z_{i}^{T} \alpha\right)-g\left(Z_{i}^{T} \alpha\right)\right] \\
=\frac{1}{\sqrt{n}} \sum_{i=1}^{n} g^{\prime}\left(Z_{i}^{T} \alpha\right) Z_{i}\left[\delta_{i}-E\left(\delta \mid Z^{T} \alpha=Z_{i}^{T} \alpha\right)\right] \\
\left.\quad \cdot \frac{1}{n} \frac{1}{f\left(Z_{i}^{T} \alpha\right) \mu\left(Z_{i}^{T} \alpha\right)} \sum_{j=1}^{n} \delta_{j} K_{h_{2}}\left(Z_{j}^{T} \alpha-Z_{i}^{T} \alpha\right)\left(\varepsilon_{j}-U_{j}^{T} \beta\right)\right\} \\
\quad-\left(\hat{\beta}_{n}-\beta\right)^{T} \frac{1}{\sqrt{n}} \sum_{i=1}^{n} g^{\prime}\left(Z_{i}^{T} \alpha\right) Z_{i}\left[\delta_{i}-E\left(\delta \mid Z^{T} \alpha=Z_{i}^{T} \alpha\right)\right] \frac{E\left(\delta X \mid Z^{T} \alpha=Z_{i}^{T} \alpha\right)}{E\left(\delta \mid Z^{T} \alpha=Z_{i}^{T} \alpha\right)} \\
\quad-\left(\hat{\alpha}_{n}-\alpha\right)^{T} \frac{1}{\sqrt{n}} \sum_{i=1}^{n} g^{\prime}\left(Z_{i}^{T} \alpha\right) Z_{i}\left[\delta_{i}-E\left(\delta \mid Z^{T} \alpha=Z_{i}^{T} \alpha\right)\right] \\
\quad \times \frac{E\left(\delta Z g^{\prime}\left(Z^{T} \alpha\right) \mid Z^{T} \alpha=Z_{i}^{T} \alpha\right)}{E\left(\delta \mid Z^{T} \alpha=Z_{i}^{T} \alpha\right)}+o_{p}(1)=J_{1}-J_{2}-J_{3}+o_{p}(1) .
\end{aligned}
$$


Similar to the second term of the left-hand side of (5.18),

$$
\begin{aligned}
J_{1}= & \frac{1}{\sqrt{n}} \sum_{i=1}^{n} \delta_{i}\left(\varepsilon_{i}-U_{i}^{T} \beta\right) \\
& \times\left\{\frac{E\left[\delta Z g^{\prime}\left(Z^{T} \alpha\right) \mid Z_{i}^{T} \alpha\right]}{E\left(\delta \mid Z_{i}^{T} \alpha\right)}-\frac{E\left[E\left(\delta \mid Z^{T} \alpha\right) Z g^{\prime}\left(Z^{T} \alpha\right) \mid Z_{i}^{T} \alpha\right]}{E\left(\delta \mid Z_{i}^{T} \alpha\right)}\right\} .
\end{aligned}
$$

Also, we have

$$
J_{2}=\sqrt{n}\left(\hat{\beta}_{n}-\beta\right)^{T} E\left\{\left[\delta-E\left(\delta \mid Z^{T} \alpha\right)\right] \frac{E\left(\delta X \mid Z^{T} \alpha\right)}{E\left(\delta \mid Z^{T} \alpha\right)} g^{\prime}\left(Z^{T} \alpha\right) Z\right\}+o_{p}(1)
$$

Combining with Lemma 5.3, we have

$$
\begin{aligned}
J_{3}= & {\left.\left[\Gamma_{\widetilde{Z}}^{-1}\left\{\frac{1}{\sqrt{n}} \sum_{i=1}^{n} \delta_{i} g^{\prime}\left(Z_{i}^{T} \alpha\right) \widetilde{Z}_{i}\left(\varepsilon_{i}-U_{i}^{T} \beta\right)-\sqrt{n} \Gamma \widetilde{Z} \widetilde{X}^{(} \hat{\beta}_{n}-\beta\right)\right\}\right]^{T} } \\
& \cdot E\left\{\left[\delta-E\left(\delta \mid Z^{T} \alpha\right)\right] \frac{E\left[\delta Z g^{\prime}\left(Z^{T} \alpha\right) \mid Z^{T} \alpha\right]}{E\left(\delta \mid Z^{T} \alpha\right)} g^{\prime}\left(Z^{T} \alpha\right) Z\right\}+o_{p}(1) .
\end{aligned}
$$

The last term of the left-hand side of (5.18) is

$$
\sqrt{n}\left(\hat{\beta}_{n}-\beta\right)^{T} E\left\{\left[(1-\delta) X-E\left((1-\delta) X \mid Z^{T} \alpha\right)\right] g^{\prime}\left(Z^{T} \alpha\right) Z\right\}+o_{p}(1)
$$

Through a direct calculation, the first term of the right-hand side of (5.18) is

$$
\sqrt{n} \Sigma \widetilde{\widetilde{Z}}\left(\breve{\alpha}_{n}-\alpha\right)+o_{p}(1)
$$

where

$$
\Sigma \widetilde{\widetilde{Z}}=E\left\{\left[\widetilde{Z} g^{\prime}\left(Z^{T} \alpha\right)\right]^{\otimes 2}\right\}
$$

The last term of the right-hand side of (5.18) is

$$
\sqrt{n} \Sigma \widetilde{\widetilde{Z}} \widetilde{\widetilde{X}_{n}}\left(\breve{\beta}_{n}-\beta\right)+o_{p}(1)
$$

where

$$
\Sigma \widetilde{\widetilde{Z}}=E\left\{\widetilde{\widetilde{Z}} g^{\prime}\left(Z^{T} \alpha\right) \widetilde{\widetilde{X}}^{T}\right\}
$$

Combining (5.19)-(5.25), and (5.27), and using Theorem 3.1, (5.18) becomes

$$
\begin{aligned}
\sqrt{n} \Sigma_{\widetilde{Z}}\left(\breve{\alpha}_{n}-\alpha\right)= & \frac{1}{\sqrt{n}} \sum_{i=1}^{n} \delta_{i}\left(\varepsilon_{i}-U_{i}^{T} \beta\right) g^{\prime}\left(Z_{i}^{T} \alpha\right) \widetilde{Z}_{i} \\
& -\frac{1}{\sqrt{n}} \sum_{i=1}^{n} \delta_{i}\left(\varepsilon_{i}-U_{i}^{T} \beta\right) g^{\prime}\left(Z^{T} \alpha\right) \frac{E\left[\left(\delta-E\left(\delta \mid Z^{T} \alpha\right)\right) Z \mid Z_{i}^{T} \alpha\right]}{E\left(\delta \mid Z_{i}^{T} \alpha\right)}
\end{aligned}
$$




$$
\begin{aligned}
& +\sqrt{n}\left(\hat{\beta}_{n}-\beta\right)^{T} E\left\{\left[\delta-E\left(\delta \mid Z^{T} \alpha\right)\right] \frac{E\left(\delta X \mid Z^{T} \alpha\right)}{E\left(\delta \mid Z^{T} \alpha\right)} g^{\prime}\left(Z^{T} \alpha\right) Z\right\} \\
& +E\left\{\left[\delta-E\left(\delta \mid Z^{T} \alpha\right)\right] \frac{E\left[\delta g^{\prime}\left(Z^{T} \alpha\right) \mid Z^{T} \alpha\right]}{E\left(\delta \mid Z^{T} \alpha\right)} g^{\prime}\left(Z^{T} \alpha\right) Z\right\}^{T} \\
& \cdot\left[\Gamma_{\widetilde{Z}}^{-1} \frac{1}{\sqrt{n}} \sum_{i=1}^{n} \delta_{i} g^{\prime}\left(Z_{i}^{T} \alpha\right) \widetilde{Z}_{i}\left(\varepsilon_{i}-U_{i}^{T} \beta\right)\right] \\
& -E\left\{\left[\delta-E\left(\delta \mid Z^{T} \alpha\right)\right] \frac{E\left[\delta Z g^{\prime}\left(Z^{T} \alpha\right) \mid Z^{T} \alpha\right]}{E\left(\delta \mid Z^{T} \alpha\right)} g^{\prime}\left(Z^{T} \alpha\right) Z\right\}^{T} \\
& \cdot\left[\Gamma_{\widetilde{Z}}^{-1} \sqrt{n} \Gamma_{\widetilde{Z} \widetilde{X}}\left(\hat{\beta}_{n}-\beta\right)\right] \\
& +\sqrt{n}\left(\hat{\beta}_{n}-\beta\right)^{T} E\left\{\left[(1-\delta) X-E\left((1-\delta) X \mid Z^{T} \alpha\right)\right] Z g^{\prime}\left(Z^{T} \alpha\right)\right\} \\
& -\Sigma_{\widetilde{Z} \widetilde{X}} \Sigma_{\widetilde{X}}^{-1}\left(\Gamma_{\widetilde{X}}+\Sigma_{1}-\Sigma_{2} \Gamma_{\widetilde{Z}}^{-1} \Gamma_{\widetilde{Z} \widetilde{X}}\right) \sqrt{n}\left(\hat{\beta}_{n}-\beta\right) \\
& +\Sigma_{\widetilde{Z} \widetilde{X}} \Sigma_{\widetilde{X}}^{-1} \Sigma_{2} \Gamma_{\widetilde{Z}}^{-1} \cdot \sqrt{n} \frac{1}{n} \sum_{i=1}^{n} \delta_{i} g^{\prime}\left(Z_{i}^{T} \alpha\right) \widetilde{Z}_{i}\left(\varepsilon_{i}-U_{i}^{T} \beta\right)+o_{p}(1) \\
& =F_{1}-F_{2}+F_{3}+F_{4}-F_{5}+F_{6}-F_{7}+F_{8}+o_{p}(1) \text {. }
\end{aligned}
$$

Through a direct calculation,

$$
\begin{aligned}
Q= & F_{1}-F_{2}+F_{4}+F_{8} \\
= & \left\{1+E\left\{\left[\delta-E\left(\delta \mid Z^{T} \alpha\right)\right] \frac{E\left[\delta Z g^{\prime}\left(Z^{T} \alpha\right) \mid Z^{T} \alpha\right]}{E\left(\delta \mid Z^{T} \alpha\right)} g^{\prime}\left(Z^{T} \alpha\right) Z\right\}^{T} \Gamma_{\widetilde{Z}}^{-1}\right. \\
& \left.+\Sigma_{\widetilde{Z} \widetilde{X}} \Sigma_{\widetilde{X}}^{-1} \Sigma_{2} \Gamma_{\widetilde{Z}}^{-1}\right\} \cdot \frac{1}{\sqrt{n}} \sum_{i=1}^{n} \delta_{i} g^{\prime}\left(Z_{i}^{T} \alpha\right) \widetilde{Z}_{i}\left(\varepsilon_{i}-U_{i}^{T} \beta\right) .
\end{aligned}
$$

Combining with Lemma 5.2, we have

$$
\begin{aligned}
P= & F_{3}-F_{5}+F_{6}-F_{7} \\
= & {\left[E\left\{\left[\delta-E\left(\delta \mid Z^{T} \alpha\right)\right] \frac{E\left(\delta X \mid Z^{T} \alpha\right)}{E\left(\delta \mid Z^{T} \alpha\right)} g^{\prime}\left(Z^{T} \alpha\right) Z\right\}^{T}\right.} \\
& -E\left\{\left[\delta-E\left(\delta \mid Z^{T} \alpha\right)\right] \frac{E\left[\delta Z g^{\prime}\left(Z^{T} \alpha\right) \mid Z^{T} \alpha\right]}{E\left(\delta \mid Z^{T} \alpha\right)} g^{\prime}\left(Z^{T} \alpha\right) Z\right\}^{T} \Gamma_{\widetilde{Z}}^{-1} \Gamma_{\widetilde{Z} \widetilde{X}} \\
& +E\left\{\left[(1-\delta) X-E\left((1-\delta) X \mid Z^{T} \alpha\right)\right] Z g^{\prime}\left(Z^{T} \alpha\right)\right\}^{T} \\
& \left.-\Sigma_{\widetilde{Z} \widetilde{X}} \sum_{\widetilde{X}}^{-1}\left(\Gamma_{\tilde{X}}+\Sigma_{1}-\Sigma_{2} \Gamma_{\widetilde{Z}}^{-1} \Gamma_{\widetilde{Z} \widetilde{X})}\right)\right] \Gamma_{\widetilde{X}}^{-1} \\
& \cdot \frac{1}{\sqrt{n}} \sum_{i=1}^{n}\left\{\delta_{i}\left[\widetilde{X}_{i}\left(\varepsilon_{i}-U_{i}^{T} \beta\right)+U_{i} \varepsilon_{i}-\left(U_{i} U_{i}^{T}-\Sigma_{u u}\right) \beta\right]\right\}+o_{p}(1) .
\end{aligned}
$$

Then, with the application of the central limit theorem, Theorem 3.2 follows immediately.

Proof of Theorem 3.3 Similar to the proof of Lemma 5.4, we first derive the asymptotical expression of $\check{g}_{n}\left(t_{0} ; \alpha, \hat{\beta}_{n}\right)$. 
From (2.8), we have

$$
\begin{aligned}
& \left(\begin{array}{c}
\check{g}_{n}\left(t_{0} ; \alpha, \check{\beta}_{n}\right) \\
h_{4} \check{g}_{n}^{\prime}\left(t_{0} ; \alpha, \check{\beta}_{n}\right)
\end{array}\right)-\left(\begin{array}{c}
g\left(t_{0}\right) \\
h_{4} g^{\prime}\left(t_{0}\right)
\end{array}\right) \\
& =\left(\frac{1}{n} B_{2}^{T} S_{2} B_{2}\right)^{-1} \frac{1}{n} \sum_{i=1}^{n} B_{i 2} K_{h_{4}}\left(t_{i}-t_{0}\right) \\
& \times\left\{\left(1-\delta_{i}\right)\left(\hat{\beta}_{n}-\beta\right)^{T} V_{i}+\left(1-\delta_{i}\right)\left[\hat{g}_{n}\left(Z_{i}^{T} \hat{\alpha}_{n}\right)-g\left(Z_{i}^{T} \alpha\right)\right]\right. \\
& \left.+\frac{1}{2}\left(\frac{t_{i}-t_{0}}{h_{4}}\right)^{2} g^{\prime \prime}\left(t_{0}\right) h_{4}^{2}+\delta_{i}\left(\varepsilon_{i}-U_{i}^{T} \beta\right)-\left(\check{\beta}_{n}-\beta\right)^{T} V_{i}\right\}+o_{p}\left(\frac{1}{\sqrt{n h_{4}}}\right) \text {. }
\end{aligned}
$$

By $\frac{h_{4}}{h_{2}} \rightarrow 0, n \rightarrow \infty$, with Lemmas 5.2-5.4, focusing on the top equation, we get

$$
\check{g}_{n}\left(t_{0} ; \alpha, \check{\beta}_{n}\right)-g\left(t_{0}\right)=\frac{1}{n} \sum_{i=1}^{n} \frac{1}{f\left(t_{0}\right)} \delta_{i} K_{h_{4}}\left(t_{i}-t_{0}\right)\left(\varepsilon_{i}-U_{i}^{T} \beta\right)+o_{p}\left(\frac{1}{\sqrt{n h_{4}}}\right) .
$$

Applying the central limit theorem, we complete the proof of Theorem 3.3.

\section{Competing interests}

The authors declare that they have no competing interests.

\section{Authors' contributions}

The authors contributed equally to the writing of this paper. All authors read and approved the final manuscript.

\section{Author details}

'College of Mathematics, Jilin University, Changchun, 130012, P.R. China. '2 Zhuhai College of Jilin University, Zhuhai, 519000, P.R. China.

\section{Acknowledgements}

The authors thank the two referees for carefully reading the paper and for their valuable suggestions and comments, which greatly improved the paper. This work is supported by National Natural Science Foundation of China (Nos. $11271155,11371168,11001105,11071126,11071269,11501241)$, Science and Technology Research Program of Education Department in Jilin Province for the 12th Five-Year Plan (440020031139) and Jilin Province Natural Science Foundation (20130101066JC, 20130522102JH, 20150520053JH, 20101596).

Received: 28 September 2015 Accepted: 9 December 2015 Published online: 05 January 2016

\section{References}

1. Ichimura, H: Estimation of single index models. Ph.D. dissertation, Department of Economics, MIT (1987)

2. Engle, RF, Granger, CWJ, Rice, J, Weiss, A: Semiparametric estimates of the relation between weather and electricity sales. J. Am. Stat. Assoc. 81, 310-320 (1986)

3. Carroll, RJ, Fan, JQ, Gijbels, I, Wand, MP: Generalized partially linear single-index models. J. Am. Stat. Assoc. 92, 477-489 (1997)

4. Yu, Y, Ruppert, D: Penalized spline estimation for partially linear single-index models. J. Am. Stat. Assoc. 97, 1042-1054 (2002)

5. Liang, H, Wang, N: Partially linear single-index measurement error models. Stat. Sin. 15, 99-116 (2005)

6. Xia, YC, Härdle, W: Semi-parametric estimation of partially linear single-index models. J. Multivar. Anal. 97, 1162-1184 (2006)

7. Xue, L, Zhu, LX: Empirical likelihood confidence regions of the parameters in a partially linear single-index model. J. R. Stat. Soc. B 68, 549-570 (2006)

8. Liu, XH, Wang, ZZ, Hu, XM: Estimation in partially linear single-index models with missing covariates. Commun. Stat., Theory Methods 41, 3428-3447 (2012)

9. Lai,, , Wang, QH: Semiparametric efficient estimation for partially linear single-index model with responses missing at random. J. Multivar. Anal. 128, 33-50 (2014)

10. Chen, $X$, Cui, HJ: Empirical likelihood for partially linear single-index errors-in-variables model. Commun. Stat., Theory Methods 38, 2498-2514 (2009)

11. Liang, H, Wang, SJ, Carrol, RJ: Partially linear models with missing response variables and error-prone covariates. Biometrika 94, 185-198 (2007)

12. Wei, $\mathrm{CH}, \mathrm{Jia}, \mathrm{XJ}, \mathrm{Hu}, \mathrm{HS}$ : Statistical inference on partially linear additive models with missing response variables and error-prone covariates. Commun. Stat., Theory Methods 44, 872-883 (2015) 
13. Wei, $\mathrm{CH}$ : Estimation of partially linear varying-coefficient errors-in-variables model with missing responses. Acta Math. Sci. 30, 1042-1054 (2010) (in Chinese)

14. Wang, QH, Sun, ZH: Estimation in partially linear models with missing responses at random. J. Multivar. Anal. 98, 1470-1497 (2007)

15. Lai, P, Wang, QH: Partially linear single-index model with missing responses at random. J. Stat. Plan. Inference 141, 1047-1058 (2011)

16. Liang, H, Härdle, W, Carroll, RJ: Estimation in a semiparametric partially linear errors-in-variables model. Ann. Stat. 15, 99-116 (1999)

17. Gai, YJ, Zhang, J, Li, GR, Luo, XC: Statistical inference on partial linear additive models with distortion measurement errors. Stat. Methodol. 27, 20-38 (2015)

18. Mark, YP, Silverman, BW: Weak and strong uniform consistency of kernel regression estimates. Z. Wahrscheinlichkeitstheor. Verw. Geb. 61, 405-415 (1982)

19. Einmahl, U, Mason, DM: Uniform in bandwidth consistency of kerneltype function estimators. Ann. Stat. 22, 1380-1403 (2005)

Submit your manuscript to a SpringerOpen ${ }^{\circ}$ journal and benefit from:

- Convenient online submission

Rigorous peer review

- Immediate publication on acceptance

- Open access: articles freely available online

- High visibility within the field

- Retaining the copyright to your article 